\title{
New options in the treatment of Cushing's disease: a focus on pasireotide
}

This article was published in the following Dove Press journal:

Research and Reports in Endocrine Disorders

28 May 2013

Number of times this article has been viewed

\author{
Anne-Gaëlle Poullot' \\ Nicolas Chevalier ${ }^{1,2}$ \\ 'Centre Hospitalier Universitaire \\ de Nice, Hôpital de l'Archet, Service \\ d'Endocrinologie, Diabétologie et \\ Médecine de la Reproduction, Nice, \\ France; ${ }^{2}$ Institut National de la \\ Santé et de la Recherche Médicale \\ (INSERM) UMR UI065/UNS, \\ Centre Méditerranéen de Médecine \\ Moléculaire (C3M), Equipe 5, \\ Environnement, Reproduction et \\ Cancers Hormono-Dépendants, \\ Nice, France
}

\begin{abstract}
Cushing's disease is caused by a corticotroph pituitary adenoma secreting adrenocorticotropin and can be fatal in the absence of adequate treatment. Transsphenoidal surgery remains the treatment of choice in almost all cases. However, remission rates are relatively low, and recurrence is usual and can be diagnosed up to decades after the initial diagnosis. Repeat surgery or radiation can be useful in these cases, although both have clear limitations with respect to efficacy and/or side effects. Hence, there is a clear unmet need for an effective medical treatment in patients with recurrent or persistent Cushing's disease. Pasireotide is a novel multireceptor-targeted somatostatin analog with a high affinity for somatostatin receptor (sstr)-1, sstr-2, sstr-3, and sstr-5. Compared with octreotide, pasireotide has an in vitro binding affinity 40 -fold higher for sstr-5, which is the major receptor subtype expressed by corticotroph pituitary adenoma. Recent studies have suggested a role for this new multireceptor somatostatin analog in Cushing's disease. We review in this article the current data available regarding pharmacokinetics, clinical efficiency, and tolerance of pasireotide in patients with de novo, persistent, or recurrent Cushing's disease, with a special focus on the disturbances of glucose metabolism induced by such a treatment.
\end{abstract}

Keywords: pasireotide, SOM230, somatostatin analog, Cushing's disease, Cushing's syndrome, pituitary adenoma, hypercortisolism

\section{Introduction}

Cushing's syndrome (CS) is a rare disorder, with an estimated incidence of 1-3 cases per million inhabitants per year. ${ }^{1,2} \mathrm{CS}$ occurs as a result of a chronic, autonomous, and pathological cortisol excess (or pathological hypercortisolism). The most common etiology of CS is the chronic administration of glucocorticoids in pharmacological doses (also called exogenous or iatrogenic CS), but it will be not considered herein.

Causes of endogenous CS can be grouped under adrenocorticotropin (ACTH)dependent and ACTH-independent categories. The first categories are due to tumorous proliferation of eutopic or ectopic cells secreting ACTH, or, very rarely, corticotropinreleasing hormone; the other categories are due to tumors of the adrenal glands that produce excess cortisol, which can be benign (adrenal adenoma) or malignant (adrenal carcinoma). ${ }^{1,2}$

The term of Cushing's disease (CD) is specifically applied to ACTH-secreting pituitary adenomas, which are the most common etiology of endogenous CS and were first described by Harvey Cushing in 1932. ${ }^{2,3}$ Excessive secretion of ACTH by a pituitary corticotropic adenoma results in excessive cortisol secretion from the adrenal glands, which can induce several clinical signs, including obesity with centripetal fat
Correspondence: Nicolas Chevalier $\mathrm{C} 3 \mathrm{M}$ and Centre Hospitalier Universitaire de Nice, $15 \mathrm{I}$ route de Saint-Antoine de Ginestière, BP 3079, 06202 Nice Cedex 3, France Tel +33492035519

Fax +33492035425

Email chevalier.n@chu-nice.fr 
disposition, signs of protein wasting (abdominal purplish skin striae, muscle weakness and atrophy, osteoporosis), high blood pressure, impaired glucose metabolism, gonadal dysfunction (libido confusion, hirsutism, menstrual irregularity) together with a broad range of psychological and psychiatric disturbances. ${ }^{4-6}$

From early reports, it is well known that CD is associated with a very poor prognosis and can be lethal. A four- to fivefold increase in mortality is classically observed in patients with remnant $\mathrm{CD}$, mostly because of cardiovascular and cerebrovascular complications and infections. ${ }^{7}$ This excess mortality is probably correlated with cortisol levels. ${ }^{7}$ Thus, the treatment goals of CD include the rapid reversal of clinical features, the normalization of cortisol levels and all biochemical changes, and long-term control without recurrence.

Transsphenoidal surgery confidently remains the first-line therapy in CD. Depending on the experience of the neurosurgeon and local invasion, it allows primary remission in $65 \%-90 \%$ of microadenomas and $50 \%-70 \%$ of macroadenomas. ${ }^{8}$ However, relapse and late recurrences of CD occur in about $25 \%$ of patients. ${ }^{9}$ As for patients for whom surgery has failed, there are limited treatment options, and this situation is probably the biggest challenge in the treatment of $\mathrm{CD}$, with no defined therapeutic approach. Second-line options are summarized in Figure 1 and include second surgery, radiotherapy, steroidogenesis inhibitors (mitotane, ketoconazole when available; metyrapone and etomidate),

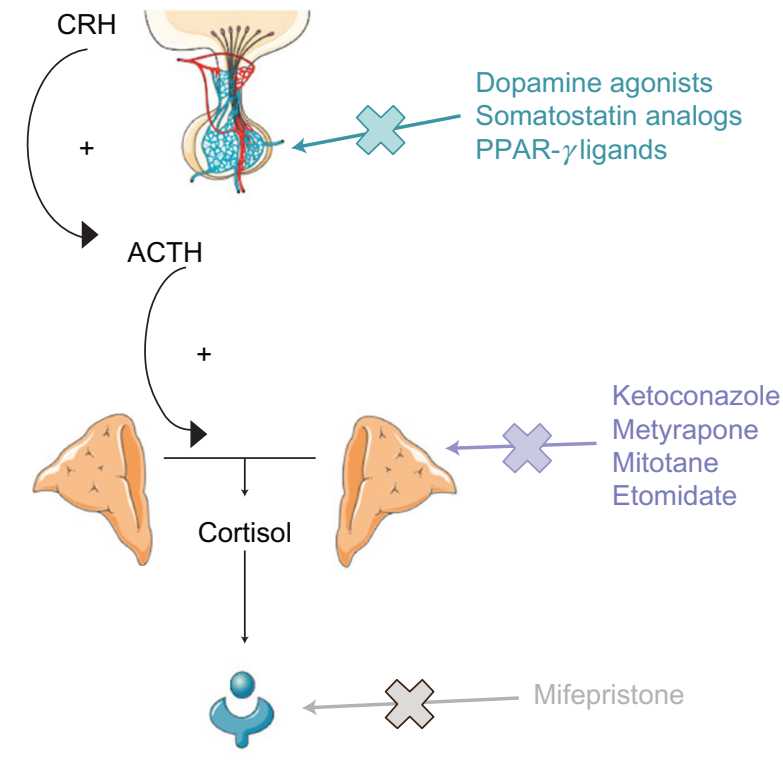

Figure I Medical treatments available for management of Cushing's disease, according to their primary site of action.

Abbreviations: $\mathrm{CRH}$, corticotropin-releasing hormone; PPAR- $\gamma$, peroxisome proliferator-activated receptor- $\gamma$; ACTH, adrenocorticotropin. glucocorticoid receptor antagonist (mifepristone), and finally bilateral adrenalectomy. ${ }^{8}$ With the exception of the last option that provides immediate control of hypercortisolism by inducing adrenal insufficiency, all of the available treatments are not very effective on $\mathrm{CD}$, with long-term remission rates lower than $50 \%$, and induce several mild or severe side effects. Therefore, there is a clear need for novel medical therapies in CD.

Unlike octreotide and lanreotide, pasireotide (SOM230 ) is a new somatostatin receptor (sstr) analog with a multireceptor affinity that can inhibit ACTH secretion. ${ }^{10}$ Pasireotide is the first drug to be approved specifically for the treatment of CD in the European Union. The present review will consider pharmacological properties of pasireotide and clinical evidence supporting its use in CD.

\section{Pharmacological aspects of pasireotide}

Because of a tight relationship between the duration of hypercortisolism and the severity of its morbidity, there is a real need for a rapid control of cortisol excess, especially in the case of immediate surgical failure or for patients who develop long-term recurrence. ${ }^{6,11}$ As previously mentioned, several alternative strategies are available. Second-line treatments are indicated in cases of surgical failure. Radiotherapy is an option, but it exhibits a slow onset of action, and patients remain exposed to the deleterious effects of cortisol excess for several years. Because of the low efficacy and the unfavorable adverse events profile of the available drugs depicted in Figure 1, the place of medical treatment is currently limited. ${ }^{5,8,10}$ Therefore, there is a clear need for medical therapies that can effectively reverse clinical features of CD by normalizing biochemical parameters acutely.

Fortunately, recent understandings in molecular physiopathology of corticotrophic adenomas have given new perspectives for the development of rational, efficacious, and safe therapy for CD. The most physiological approach is to directly act on the ACTH hypersecretion source by using a pituitary-targeted therapy, with antisecretory and, if possible, antiproliferative effects. Because of the presence of subtype 2 dopamine receptors and sstr on corticotroph adenomas, dopamine agonists and sstr agonists have been carefully screened for a potential use in CD. ${ }^{12}$

Somatostatins (also known as somatotropin-release inhibitory factors) affect the release of many hormones, and thus are important regulators of endocrine secretion in many tissues. Somatostatin binds with high affinity to five distinct G-protein-coupled sstr (sstr-1 to -5), expressed on 
target tissues, which differ in their binding properties and pharmacology. ${ }^{13}$ Human corticotropic pituitary adenomas express multiples sstr, but predominantly sstr-5 (90\%), sstr-2 (70\%), and sstr-1 (60\%). ${ }^{14}$ Several studies reported that both sstr-2 and sstr-5 ligands could inhibit corticotropin-releasing hormone-stimulated ACTH secretion in mouse or human corticotropic cells. Furthermore, activation of sstr-5 subtype inhibits ACTH secretion, providing a potential therapeutic target in the treatment of CD. ${ }^{15,16}$

Unfortunately, natural somatostatins (SRIF-14, SRIF28) exhibit a high binding affinity for all sstr subtypes, but their therapeutic use is mainly limited because of their rapid degradation in plasma. ${ }^{13}$ Furthermore, the two synthetic somatostatin analogs approved for clinical use, octreotide (SMS 201-995) and lanreotide (BIM 23014), bind preferentially with a high affinity to the sstr-2 subtype and exhibit no effect on ACTH secretion. ${ }^{13}$ Recently, a new synthetic somatostatin analog has been relapsed. Pasireotide (SOM-230) is a small, metabolically stable somatostatin analog with a more universal receptor-binding profile, closer to that of natural somatostatins. It exhibits a highaffinity binding to four of the five receptor subtypes (sstr1, sstr-2, sstr-3, and sstr-5), with the highest affinity for sstr-5. ${ }^{13}$ Compared with octreotide and lanreotide, major improvements have been achieved, especially with regard to sstr-1 and sstr-5 binding, because pasireotide has an in vitro binding affinity 20-30 times higher for sstr-1, and 40-100 times higher for sstr-5 than octreotide and lanreotide exhibit, respectively (Figure 2). Interestingly, pasireotide demonstrates a binding affinity to sstr-5 that is even higher

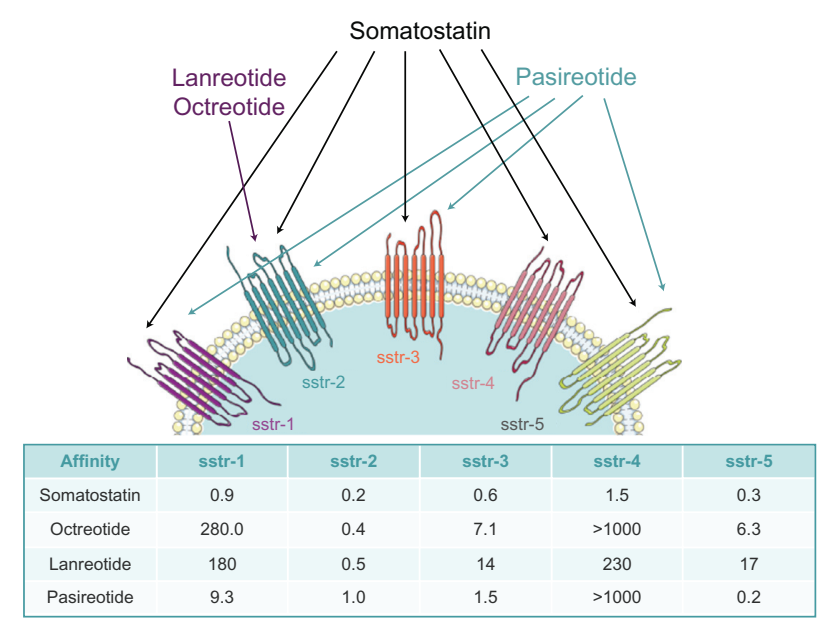

Figure 2 Schematic representation of preferential binding affinities of somatostatin, classical somatostatin analogs (lanreotide and octreotide), and pasireotide for the five known somatostatin receptor subtypes (sstr-I to sstr-5).

Note: Results are expressed as mean values (in nanomoles per liter).

Abbreviation: sstr, somatostatin receptor. than the affinity measured for the natural ligand SRIF-14 for this receptor subtype. ${ }^{13}$

Mouse corticotropic pituitary adenoma cells show in vitro a high ribonucleic acid (RNA) expression of sstr-5 receptors but a relatively closed RNA expression of sstr- 2 receptors, reflecting the expression profile of human corticotropic adenomas. ${ }^{17}$ Suppressive effects of somatostatin analogs in corticotropic cells are determined by sstr-5, whereas the ligand action on sstr-2 is negligible, explaining the inefficiency of octreotide and lanreotide in CD. ${ }^{18}$ In primary cultures of mouse corticotropic adenoma cells, pasireotide is highly effective in lowering basal and stimulated ACTH release. ${ }^{15,16}$ Besides, pretreatment by dexamethasone does not affect the inhibitory effect of pasireotide on these cells, suggesting that sstr-5 is relatively resistant to downregulation by glucocorticoid treatment (and thus by elevated endogenous cortisol levels). ${ }^{15,16}$ In addition, significant inhibition of cell proliferation has been recently reported in vitro in primary cell culture and in vivo in rats; pasireotide affects only cell division but has no effect on cell differentiation. ${ }^{19}$

The pharmacokinetic profile of pasireotide has been determined in vivo by several studies conducted on rats. Its distribution and terminal elimination half-life are 0.74 hours and 23 hours, respectively, which are considerably longer than the corresponding values of 0.22 hours and 2.0 hours determined for octreotide (three- and elevenfold longer, respectively). ${ }^{13}$ Compared with control, the strong inhibition of the hypothalamic-pituitary-adrenal axis by pasireotide has been confirmed, with a $45 \%-51 \%$ inhibitory effect on ACTH release. ${ }^{20}$

Referring to these promising results, several Phase I studies have followed to investigate the safety, tolerability, and pharmacokinetic profile of pasireotide in human subjects. In healthy volunteers, the effective terminal half-life is $12-17$ hours after a single-dose administration of 900-1500 $\mu \mathrm{g}$, supporting a twice-daily dosing interval in therapeutic use. ${ }^{21}$ Pasireotide also demonstrates rapid absorption, low systemic clearance, a high volume of distribution, and a dose-proportionally effect. The tolerance and safety profile of pasireotide is quite similar to other somatostatin analogs, including mild gastrointestinal adverse events, injection-site reactions, but more frequently hyperglycemia. ${ }^{21}$

\section{Clinical evidence}

In order to demonstrate the efficiency of pasireotide treatment, a Phase II, proof-of-concept, open-label, single-arm, multicenter study was driven. ${ }^{22}$ Over 15 days, 
patients with de novo, persistent, or recurrent $\mathrm{CD}$, recruited from ten centers, self-administered subcutaneous pasireotide $600 \mu \mathrm{g}$ twice daily and primary efficacy outcome was evaluated considering normalization of mean urinary free cortisol (UFC) levels, defined as the mean of two 24-hour urine specimens. Secondary endpoints were evaluated by assessing changes in plasma ACTH and serum cortisol. Evaluation of primary efficacy was possible in 29 of the 39 included patients; five of them (17\%; 95\% confidence interval $[\mathrm{CI}]=6 \%-36 \%$ ) achieved a normal UFC level after 15 days of treatment. Overall, the mean UFC level decreased from baseline by $44.5 \%$ (from $1231 \pm 1141 \mathrm{nmol} / 24$ hours to $683 \pm 615 \mathrm{nmol} / 24$ hours $; P=0.021)$. UFC levels decreased in 22 of 29 patients $(76 \%)$. Although a significant correlation between baseline UFC level and UFC level at day 15 was not observed, there was a trend toward a lower baseline UFC level being predictive of a response to pasireotide $(P=0.102)$. At baseline, the mean level of serum cortisol was $5046 \mathrm{nmol} \cdot \mathrm{h} / \mathrm{L}$ (area under the curve [AUC] 0-8 hour serum cortisol). There was no significant difference between responders and nonresponders based on values at baseline or at the end of the study. However, a significant difference was observed in serum cortisol reduction in responders compared with nonresponders $(1248 \pm 990 \mathrm{nmol} \cdot \mathrm{h} / \mathrm{L}$ versus $420 \pm 932 \mathrm{nmol} \cdot \mathrm{h} / \mathrm{L} ; P=0.04)$. Considering ACTH levels, the mean AUC 0-8 hours of plasma ACTH was $123 \mathrm{pmol} \cdot \mathrm{h} / \mathrm{L}$ at baseline. There was neither a significant difference between responders and nonresponders based on baseline values or values at the end of the study, nor a significant difference in ACTH reduction between responders and nonresponders. Responders exhibited an approximately 1.8-fold higher plasma concentration $\left(\mathrm{C}_{\min }\right)$ and a 1.3-fold higher plasma exposure (AUC 0-8 hours) to pasireotide, despite similar $\mathrm{C}_{\max }$ values. Therefore, these results suggested that clinical response may be related to pasireotide plasma exposure. ${ }^{10,22}$

Based on these positive results, a large international, double-blinded, randomized Phase III trial was assessed. ${ }^{23}$ This largest Phase III study in patients with CD compared subcutaneous pasireotide $600 \mu \mathrm{g}$ and $900 \mu \mathrm{g}$ twice-daily injections in patients with de novo, persistent, or recurrent CD, defined by: (1) a mean 24-hour UFC level greater than 1.5 upper limit of the normal range (ULN), calculated from four 24-hour samples collected within 2 weeks; (2) a morning plasma ACTH level of $5 \mathrm{ng} / \mathrm{L}$ or more, and (3) a confirmed pituitary adenoma. After 3 and 6 months of treatment, if the mean 24-hour UFC was not controlled, patients were unblinded and the dose increased by $300 \mu \mathrm{g}$ twice daily, with a maximum of $1200 \mu \mathrm{g}$ twice daily. Among the 162 included patients, 78 (48\%) completed 12 months of treatment, with a mean duration of treatment of 10.8 months.

The mean baseline level of the 24-hour UFC was approximately 6.5 times the ULN range. After 6 months of treatment, $88 \%$ of the evaluable patients (91/103) had a decline in 24-hour UFC, including patients with very high baseline values. Normalization of 24-hour UFC was achieved in $26.3 \%(21 / 80)$ and $14.6 \%$ (12/82) of patients receiving the higher and lower doses, respectively. A normal UFC level was mainly achieved in patients with baseline levels not exceeding five times the ULN than in patients with higher baseline levels. The lower remission rate in the $600 \mu \mathrm{g}$ twice-daily group may in part be explained by the worse mean UFC levels at the baseline compared with those in the $900 \mu \mathrm{g}$ twice-daily group (1156 nmol/24 hours versus $782 \mathrm{nmol} / 24$ hours). However, the mean percentage change in the UFC level from baseline to month 6 was $-27.5 \%$ $(95 \% \mathrm{CI}=-55.9 \%$ to $0.9 \%)$ in the $600 \mu \mathrm{g}$ group and $-48.4 \%$ $(95 \% \mathrm{CI}=-56.6 \%$ to $-40.2 \%)$ in the $900-\mu \mathrm{g}$ group. The corresponding changes from baseline to month 12 were $-41.3 \%(95 \% \mathrm{CI}=-66.0 \%$ to $-16.6 \%)$ and $-54.5 \%$ $(95 \%$ CI $=-65.2 \%$ to $-43.7 \%)$, respectively. The change from baseline to months 6 and 12 was significant for both groups ( $P<0.001)$. Partial control of hypercortisolism, defined as a UFC level above the ULN but reduced by more than $50 \%$ from baseline, was achieved in $18 \%$ and $13 \%$ of patients in the $600 \mu \mathrm{g}$ and $900 \mu \mathrm{g}$ groups at month 6 , and in $16 \%$ and $3 \%$ at month 12 , respectively. UFC levels decreased quickly, with a median reduction of approximately $50 \%$ at month 2 , and remained stable in both groups, indicating a prolonged therapeutic benefit.

Reduction in biochemical parameters in response to pasireotide was mild ( $-13.4 \%$ for morning serum cortisol and $-16.9 \%$ for plasma ACTH level at month 12), but strongly improved clinical features of $\mathrm{CD}$, with a decrease of systolic and diastolic blood pressure $(-6.1 \mathrm{mmHg}$ and $-3.7 \mathrm{mmHg}$, respectively), triglycerides $(-0.2 \mathrm{mmol} / \mathrm{L})$, low-density lipoprotein cholesterol $(-0.4 \mathrm{mmol} / \mathrm{L})$, weight $(-6.7 \mathrm{~kg})$, and an amelioration of health-related quality-oflife score ( +11.1 points).

Interestingly, a lack of response could be identified quite quickly in patients. Indeed, among the 72 patients with uncontrolled hypercortisolism at the first 2 months, hypercortisolism remained uncontrolled in 66 patients (92\%) at month 6 and in 64 patients (89\%) at month 12. Thus, efficiency of pasireotide can be evaluated after 2-3 months of treatment, and if normalization of UFC levels is not achieved, 
combination therapy or alternative treatment modalities should be considered.

Finally, like other somatostatin analogs, pasireotide participated in tumor shrinkage, with an estimated decrease of tumoral volume of $9.1 \%$ (95\% CI $=-46.3 \%$ to $28.0 \%)$ and $43.8 \%(95 \% \mathrm{CI}=-68.4 \%$ to $-19.2 \%)$ in the $600 \mu \mathrm{g}$ and the $900 \mu \mathrm{g}$ twice-daily groups, respectively. ${ }^{23}$

\section{Tolerance profile of pasireotide}

In different studies, the safety profile of pasireotide is quite similar to that of all somatostatin analogs. ${ }^{10,21-23}$ Most frequent adverse events are gastrointestinal symptoms (diarrhea, nausea, abdominal pain, cholelithiasis) and vascular disorders (flush, hypotension). Most of them resolve spontaneously without dose modification or temporary interruption. Mild transient elevations of liver enzyme activity are also reported in $29 \%$ of patients, with a spontaneous resolution in most cases without treatment interruption. ${ }^{23}$

However, unlike other somatostatin analogs, pasireotide determines more frequent and more severe glucose homeostasis troubles (36\% in Phase II and 73\% in Phase III, respectively). ${ }^{22,23}$ This potential diabetogenic profile of pasireotide is important to take into account because glucose tolerance is already impaired in about $50 \%$ of patients with $\mathrm{CD}$ and participates in the excess mortality. ${ }^{1,8}$ Indeed, glucocorticoids impair glucose regulation through several mechanisms, including insulin resistance and pancreatic $\beta$-cell function impairment. ${ }^{24}$ Glucocorticoids interfere with $\beta$-cell metabolism by decreasing the expression of glucose transporter 2 and glucokinase, leading to a defective exocytosis of insulin granules. ${ }^{25}$ In liver, glucocorticoids enhance neoglucogenesis and antagonize glycogen synthesis. ${ }^{26}$ They also reduce the insulinotropic effect of incretin hormones, particularly glucagon-like peptide 1 (GLP-1). ${ }^{27}$ Finally, glucocorticoids have deleterious effects on the insulin sensitivity of target tissues and promote the metabolic phenotype (visceral fat accumulation). ${ }^{26}$

Data obtained in healthy volunteers receiving pasireotide suggest that pasireotide-induced hyperglycemia is a result of decreased insulin and incretin secretion, whereas insulin sensitivity is unaffected. ${ }^{28}$ Indeed, a single subcutaneous dose of pasireotide leads to a rapid, dose-dependent transient increase of blood-glucose levels. At the same time, mean plasma GLP-1 levels are reduced, as well as insulin and glucagon mean values, with a greater suppression of insulin secretion than glucagon secretion. Normalization of glucose levels is achieved in about 10 hours after pasireotide injection. These data are confirmed during $75 \mathrm{~g}$ oral glucose tolerance. Pasireotide-induced hyperglycemia could therefore be mediated by a severe defect in the secretion of insulin and incretin hormones (GLP-1). ${ }^{28}$

In fact, because the presence of sstr on $\alpha$ - and $\beta$-pancreatic cell membranes, natural somatostatin is able to inhibit $\beta$-cell insulin secretion through sstr-2 and sstr-5 receptor subtypes and $\alpha$-cell glucagon secretion through the sstr- 2 receptor subtype. Because of their predominantly binding affinity for the sstr-2 receptor subtype, the first-generation somatostatin analogs octreotide and lanreotide have less detrimental effect on insulin/glucagon balance than does pasireotide, which inhibits insulin secretion much more potently than glucagon because of its high affinity for sstr-5 (Figure 3 ). These findings suggest that the ratio of sstr-5/sstr- 2 is the major factor in pasireotide-induced hyperglycemia. ${ }^{29,30}$

In a Phase II study, hyperglycemia was observed in $36 \%$ of patients $(14 / 39)$ and appeared to be more notable in patients with a history of impaired fasting blood glucose or diabetes mellitus before receiving pasireotide. ${ }^{22}$ Because of the suppression of insulin secretion, hyperglycemia occurred just after the first exposure to pasireotide but generally improved to near baseline value in 8 hours. It was less pronounced after a few days of treatment, suggesting an attenuation of effect over time.

In a Phase III study, hyperglycemia was reported in 73\% of patients (118/162) and led to treatment discontinuation in $6 \% .{ }^{23}$ Preexisting diabetes mellitus or impaired glucose tolerance increased the risk of hyperglycemia-related adverse events. Fasting blood glucose and glycated hemoglobin levels increased soon after the initiation of treatment with pasireotide, and then stabilized after 2 months. Hemoglobin $\mathrm{A}_{1 \mathrm{c}}\left(\mathrm{HbA}_{1 \mathrm{c}}\right)$

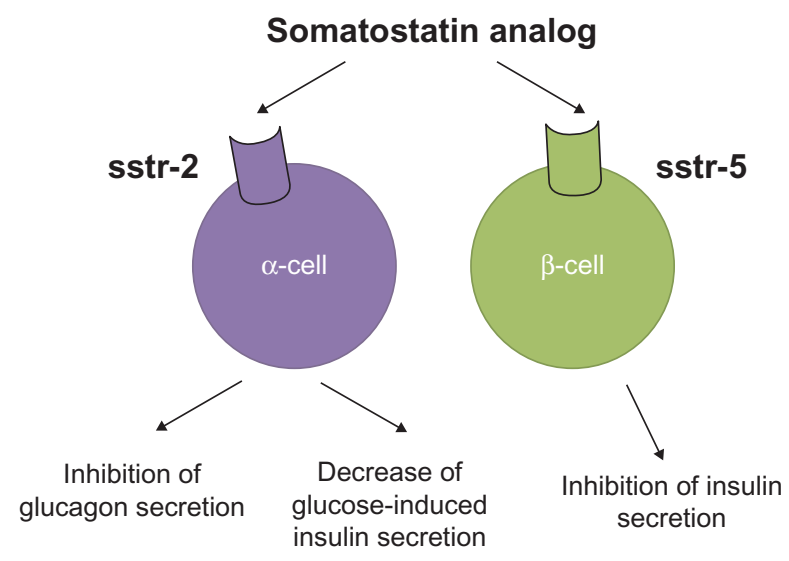

Figure 3 Schematic representation of the effects of somatostatin analogs on $\alpha$ - and $\beta$-pancreatic cells through sstr- 2 and sstr- 5 receptors.

Note: Because of its predominantly binding affinity for the sstr-5 receptor subtype, pasireotide has more detrimental effects on insulin/glucagon balance than do octreotide and lanreotide.

Abbreviation: sstr, somatostatin receptor. 
increased from $5.8 \%$ to $7.2 \%$ and $7.4 \%$ after 6 months of treatment, and to $7.3 \%$ and $7.2 \%$ after 12 months of treatment with pasireotide $600 \mu \mathrm{g}$ and $900 \mu \mathrm{g}$ twice daily, respectively. Considering an $\mathrm{HbA}_{1 \mathrm{c}}$ level $>6.5 \%, 48 \%$ of the patients receiving pasireotide (51/107) developed a new-onset diabetes mellitus and $41 \%$ needed a specific treatment with one or more antidiabetic medications. No case of diabetic ketoacidosis or hyperosmolar hyperglycemia was reported.

Interestingly, pasireotide-induced hyperglycemia occurred despite declining cortisol levels. In one patient, the worsening role of pasireotide on glycemic condition has been minimized by the resolution of hypercortisolism status. ${ }^{31}$ Thus, it seems legitimate to affirm that the effects of pasireotide on hormonal adjustment prevail on its adverse effects, especially because glucose-lowering therapy may be quite effective. Nevertheless, only few data are available concerning treatment strategy for pasireotide-induced hyperglycemia, and none of them have been evaluated in randomized controlled trials. Recently, French recommendations for the managing of pasireotide-induced hyperglycemia in CD have been proposed using the clinical data available: ${ }^{32}$ (1) initial monitoring of blood glucose and $\mathrm{HbA}_{1 \mathrm{c}}$ levels, especially in patients with preexisting diabetes mellitus, impaired glucose tolerance or risk factors of diabetes mellitus; (2) fasting plasma glucose monitoring 1, 2, 4, 8, and 12 weeks after initiating pasireotide, and 1, 2, and 4 weeks after each dose regimen modification; (3) self-monitoring of blood glucose at least once or twice a week in fasting and postprandial states; and (4) monitoring of fasting plasma glucose and $\mathrm{HbA}_{\mathrm{lc}}$ after treatment discontinuation in patients exhibiting glucose tolerance abnormalities. Expert recommendations suggest that patients with uncontrolled diabetes mellitus, as indicated by a recent history of ketoacidosis or $\mathrm{HbA}_{1 \mathrm{c}}>8 \%$, should not be treated with pasireotide. In other situations, the initiation or intensification of antidiabetic therapy should be performed as soon as possible. ${ }^{32}$

Concerning therapeutic strategy, the only data available concern the short-term effects of antidiabetic drugs used in healthy volunteers exposed to pasireotide. Interestingly, despite the insulin resistance observed in $\mathrm{CD}$, metformin seems to have little effect on glycemic excursions, whereas such excursions are improved by nateglinide, vildagliptin, and liraglutide. ${ }^{28}$ Of course, therapeutic strategy should always consider individual risk-benefit ratio, and be individually adapted, especially using data obtained from blood glucose self-monitoring. However, if hyperglycemia remains uncontrolled despite appropriate antidiabetic measures, pasireotide therapy should be adapted with dose reduction, or even discontinued, depending on the risk-benefit balance and possible alternative treatments for hypercortisolism. ${ }^{32}$ These recommendations are supported by similar recent proposals developed from a group of ten European experts in the field of pituitary diseases and diabetes mellitus, which provide initial therapy with metformin followed by the addition of a dipeptidyl peptidase-4 inhibitor, with a switch from the dipeptidyl peptidase 4 inhibitor to a GLP-1 receptor agonist or insulin therapy in order to maintain glycemic targets. ${ }^{33}$

\section{Prospects and conclusion}

It is now well demonstrated that pasireotide is able to provide significant clinical benefit in patients with $\mathrm{CD}$ by reducing hypercortisolism and improving associated signs and symptoms. Pasireotide may represent the first specific treatment for corticotropic adenomas. However, more data about long-term experience with pasireotide are required in order to strengthen the known results.

The Phase III study, mentioned on the previous page, was extended to 24 months to evaluate the long-term effect of pasireotide treatment. ${ }^{34}$ Fifty-eight patients entered into the extension phase and sustained a decrease in mean UFC, serum cortisol, and plasma ACTH already observed through to month 24. Furthermore, control of UFC was maintained up to month 24 in $34.5 \%$ of patients, without new serious adverse events.

Recently, a French team reported the case of a 43-yearold woman treated with pasireotide for 7 years. ${ }^{35}$ Initially, her 24-hour UFC levels were 9.2 times the ULN, and she began 15 days of treatment with subcutaneous pasireotide (600 $\mu \mathrm{g}$ twice daily), which resulted in normalization of these levels. When treatment was halted for 35 days, UFC levels increased anew. Two months later, she resumed treatment with pasireotide $600 \mu \mathrm{g}$ twice daily, which led to significant and prolonged clinical improvement. To date, UFC levels have remained in the normal range, as well as basal and desmopressin-stimulated ACTH levels, without any serious adverse events.

These data illustrate the clear biochemical and clinical response that can be awaited in long-term treatment with pasireotide in patients with $\mathrm{CD}$, without any treatment escape. Achieving a long-acting formulation of pasireotide, leading to a unique monthly intramuscular injection, is a new challenge and would be a significant progress in improving the quality of life of patients.

\section{Acknowledgments}

The authors contributed equally to this review. They wrote, read, and approved the final version of the manuscript. 


\section{Disclosure}

$\mathrm{N}$ Chevalier carried out clinical trials as coinvestigator for Novartis and Ipsen, has received speaker's fees from Ipsen, and has also received research grants from Novartis. The authors report no other conflicts of interest in this work.

\section{References}

1. Castinetti F, Morange I, Conte-Devolx B, Brue T. Cushing's disease. Orphanet J Rare Dis. 2012;7:41.

2. Bertagna X, Guignat L, Groussin L, Bertherat J. Cushing's disease. Best Pract Res Clin Endocrinol Metab. 2009;23(5):607-623.

3. Cushing H. The basophil adenomas of the pituitary body and their clinical manifestations. Pituitary basophilism. Bull Johns Hopkins Hosp. 1932;50(4):137-195.

4. Nieman LK, Biller BM, Findling JW, et al. The diagnosis of Cushing's syndrome: an Endocrine Society Clinical Practice Guideline. J Clin Endocrinol Metab. 2008;93(5):1526-1540.

5. Newell-Price J, Bertagna X, Grossman AB, Nieman LK. Cushing's syndrome. Lancet. 2006;367(9522):1605-1617.

6. Findling JW, Raff H. Cushing's Syndrome: important issues in diagnosis and management. J Clin Endocrinol Metab. 2006;91(10):3746-3753.

7. Dekkers OM, Biermasz NR, Pereira AM, et al. Mortality in patients treated for Cushing's disease is increased, compared with patients treated for nonfunctioning pituitary macroadenoma. J Clin Endocrinol Metab. 2007;92(3):976-981.

8. Biller BM, Grossman AB, Stewart PM, et al. Treatment of adrenocorticotropin-dependent Cushing's syndrome: a consensus statement. J Clin Endocrinol Metab. 2008;93(7):2454-2462.

9. Patil CG, Prevedello DM, Lad SP, et al. Late recurrences of Cushing's disease after initial successful transsphenoidal surgery. $J$ Clin Endocrinol Metab. 2008;93(2):358-362.

10. Pedroncelli AM. Medical treatment of Cushing's disease: somatostatin analogues and pasireotide. Neuroendocrinology. 2010;92 Suppl 1: $120-124$

11. Arnaldi G, Angeli A, Atkinson AB, et al. Diagnosis and complications of Cushing's syndrome: a consensus statement. J Clin Endocrinol Metab. 2003;88(12):5593-5602.

12. de Bruin C, Feelders RA, Lamberts SW, Hofland LJ. Somatostatin and dopamine receptors as targets for medical treatment of Cushing's Syndrome. Rev Endocr Metab Disord. 2009;10(2):91-102.

13. Bruns C, Lewis I, Briner U, Meno-Tetang G, Weckbecker G. SOM230: a novel somatostatin peptidomimetic with broad somatotropin release inhibiting factor (SRIF) receptor binding and a unique antisecretory profile. Eur J Endocrinol. 2002;146(5):707-716.

14. van der Hoek J, Lamberts SW, Hofland LJ. Preclinical and clinical experiences with the role of somatostatin receptors in the treatment of pituitary adenomas. Eur J Endocrinol. 2007;156 Suppl 1:S45-S51.

15. Hofland LJ, van der Hoek J, Feelders R, et al. The multi-ligand somatostatin analogue SOM230 inhibits ACTH secretion by cultured human corticotroph adenomas via somatostatin receptor type 5. Eur J Endocrinol. 2005;152(4):645-654.

16. van der Hoek J, Waaijers M, van Koetsveld PM, et al. Distinct functional properties of native somatostatin receptor subtype 5 compared with subtype 2 in the regulation of ACTH release by corticotroph tumor cells. Am J Physiol Endocrinol Metab. 2005;289(2):E278-E287.

17. Schmid HA, Silva AP. Short- and long-term effects of octreotide and SOM230 on GH, IGF-I, ACTH, corticosterone and ghrelin in rats. J Endocrinol Invest. 2005;28(Suppl International 11):28-35.

18. Ben-Shlomo A, Schmid H, Wawrowsky K, et al. Differential ligandmediated pituitary somatostatin receptor subtype signaling: implications for corticotroph tumor therapy. J Clin Endocrinol Metab. 2009;94(11): $4342-4350$.
19. Nolan LA, Schmid HA, Levy A. Octreotide and the novel multireceptor ligand somatostatin receptor agonist pasireotide (SOM230) block the adrenalectomy-induced increase in mitotic activity in male rat anterior pituitary. Endocrinology. 2007;148(6):2821-2827.

20. Silva AP, Schoeffter P, Weckbecker G, Bruns C, Schmid HA. Regulation of $\mathrm{CRH}$-induced secretion of ACTH and corticosterone by SOM230 in rats. Eur J Endocrinol. 2005;153(3):R7-R10.

21. Petersenn S, Hu K, Maldonado M, et al. Tolerability and dose proportional pharmacokinetics of pasireotide administered as a single dose or two divided doses in healthy male volunteers: a single-center, open-label, ascending-dose study. Clinical Ther. 2012;34(3):677-688.

22. Boscaro M, Ludlam WH, Atkinson B, et al. Treatment of pituitarydependent Cushing's disease with the multireceptor ligand somatostatin analog pasireotide (SOM230): a multicenter, phase II trial. J Clin Endocrinol Metab. 2009;94(1):115-122.

23. Colao A, Petersenn S, Newell-Price J, et al; for Pasireotide B2305 Study Group. A 12-month phase 3 study of pasireotide in Cushing's disease. N Engl J Med. 2012;366(10):914-924.

24. Mazziotti G, Gazzaruso C, Giustina A. Diabetes in Cushing syndrome: basic and clinical aspects. Trends Endocrinol Metab. 2011;22(12): 499-506.

25. van Raalte DH, Ouwens DM, Diamant M. Novel insights into glucocorticoid-mediated diabetogenic effects: towards expansion of therapeutic options? Eur J Clin Invest. 2009;39(2):81-93.

26. Pivonello R, De Leo M, Vitale P, et al. Pathophysiology of diabetes mellitus in Cushing's syndrome. Neuroendocrinology. 2010;92 Suppl 1: $77-81$.

27. Hansen KB, Vilsbøll T, Bagger JI, Holst JJ, Knop FK. Reduced glucose tolerance and insulin resistance induced by steroid treatment, relative physical inactivity, and high-calorie diet impairs the incretin effect in healthy subjects. J Clin Endocrinol Metab. 2010;95(7):3309-3317.

28. Henry RR, Mudaliar S, Wetli-Hermosillo K, Ligueros-Saylan M, Chenji S, Golor G. Mechanism and management of hyperglycemia associated with pasireotide: results from studies in healthy volunteers. Endocrine Rev. 2011;32:P3-274.

29. Zambre Y, Ling Z, Chen MC, et al. Inhibition of human pancreatic islet insulin release by receptor-selective somatostatin analogs directed to somatostatin receptor subtype 5. Biochem Pharmacol. 1999;57(10): 1159-1164.

30. Singh V, Brendel MD, Zacharias S, et al. Characterization of somatostatin receptor subtype-specific regulation of insulin and glucagon secretion: an in vitro study on isolated human pancreatic islets. J Clin Endocrinol Metab. 2007;92(2):673-680.

31. Fiorentino C, Motta C, Wolosinska DT, Monti S, Mori F, Toscano V. SOM230 in Cushing's disease complicated by poorly controlled diabetes mellitus. J Endocrinol Invest. 2011;34(9):731-732.

32. Reznik Y, Bertherat J, Borson-Chazot F, et al. Management of hyperglycaemia in Cushing's disease: experts' proposals on the use of pasireotide. Diabetes Metab. 2013;39(1):34-41.

33. Colao A, De Block C, Gaztambide MS, Kumar S, Seufert J, Casanueva FF. Managing hyperglycemia in patients with Cushing's disease treated with pasireotide: medical expert recommendations. Pituitary. Epub April 7, 2013.

34. Pivonello R, van Gaal L, Salgado LR, et al. Long-term use of pasireotide results in sustained reductions in UFC and continued improvements in signs and symptoms in patients with Cushing's disease. Proceedings of the 15th Congress of the European Neuroendocrine Association; September 12-15, 2012; Vienna, Austria.

35. Libé R, Groussin L, Bertherat J. Pasireotide in Cushing's disease. N Engl J Med. 2012;366(22):2134; author reply 2134-2135. 
Research and Reports in Endocrine Disorders

Dovepress

\section{Publish your work in this journal}

Research and Reports in Endocrine Disorders is an international, peerreviewed, open access journal publishing original research, reports, reviews and commentaries on all areas of endocrinology, endocrine disorders and therapeutic interventions. The manuscript management system is completely online and includes a very quick and fair to read real quotes from published authors.

Submit your manuscript here: http://www.dovepress.com/research-and-reports-in-endocrine-disorders-journal 